\title{
Radiaton Effects on TAC-PF Electromagnetic Calorimeter
}

\author{
E. Pilicer*, F. Kocak, A. Kilic, F.B. Pilicer and I. Tapan \\ Uludag University, Department of Physics, 16059 Bursa, Turkey
}

\begin{abstract}
The proposed particle factory detector for Turkish Accelerator Center (TAC-PF) being a regional facility in Turkey will operate at the center of mass energy about $3.77 \mathrm{GeV}$ with designed luminosity of the order of $10^{34} \mathrm{~cm}^{-2} \mathrm{~s}^{-1}$. The electromagnetic calorimeter part of the detector is considered to have $\mathrm{PbWO}_{4}$ and $\mathrm{CsI}(\mathrm{Tl})$ crystals coupled with photodiodes. In this study, the exposed dose rate to electromagnetic calorimeter during PF detector operation was estimated by FLUKA Monte Carlo tool. The irradiation effects such as the change of light yield and light transmission of these crystals were investigated to evaluate TAC-PF electromagnetic calorimeter energy resolution.
\end{abstract}

DOI: 10.12693/APhysPolA.131.190

PACS/topics: 42.88.+h, 07.20.Fw, 02.70.Uu

\section{Introduction}

The Turkish Accelerator Center (TAC) [1] project was considered as a regional accelerator facility for fundamental and applied physics research in Turkey. The electronpositron collider comprises of a super charm factory consisting of a $1 \mathrm{GeV}$ electron linac and a $3.6 \mathrm{GeV}$ positron ring for linac on ring type [2] and a detector to search for charm physics, mainly the $\mathrm{CP}$ violation and mixings of $D^{0}$ mesons, as well as new physics effects in the rare decays and is one of the main parts of the TAC project. The main components of the TAC Particle Factory (TAC-PF) detector are tracker $(\mathrm{SiT})$, time of flight (ToF), calorimeter (ECAL), and muon system (MUON). The aim of this paper is to investigate the radiation damage effects to the electromagnetic energy resolution.

Radiation has crucial effects on the detector performance during its operation. Whenever secondary particles released from the collisions have sufficiently large energy to displace the atoms from lattice, the detector starts to be degraded and damaged. The radiation dose rate is important parameter determining the amount of radiation damage and it reaches saturation of the damage after a small-integrated dose.

The predominant radiation damage is the radiation induced light absorption because of the formation of color centers, which are reduced light attenuation length and thus light output and also may degrade light response uniformity of crystal scintillators. The other damage mechanism is the phosphorescence, which leads to an increase in the readout noise. The last one is the damage of scintillation mechanism. This damage can reduce the scintillation light yield, which leads to a decrease in the light output and the distortion of light response uniformity. The variations of the light attenuation length due to the given radiation dose leads to the degradation of transmittance of crystal scintillators and the decrease

*corresponding author; e-mail: epilicer@uludag.edu.tr of light output. Non-uniformity of light output along the crystal leads to an unrecoverable deterioration in the energy resolution [3].

In the TAC-PF ECAL, we have considered both Bogoroditsk Plant of Technochemical Products (BTCP)$\mathrm{PbWO}_{4}$ (PWO) and Shanghai Institute of Ceramics (SIC)-CsI(Tl) crystals coupled to either avalanche photodiode (APD) or PIN photodiodes. Each crystal has been arranged in $5 \times 5$ matrices. PWO crystal has a dimension of $20 \times 20 \times 200 \mathrm{~mm}^{3}\left(22 X_{0}\right)$, while $\mathrm{CsI}(\mathrm{Tl})$ crystal has a dimension of $55 \times 55 \mathrm{~mm}^{2}$ at the front face and $60 \times 60 \mathrm{~mm}^{2}$ at the rear face with a length of $300 \mathrm{~mm}$ $\left(16.2 X_{0}\right)$ as in Ref. [4]. Changes in some properties of PWO and $\mathrm{CsI}(\mathrm{Tl})$ crystals after irradiation are shown in Table I. For the photodetectors, we have considered Hamamatsu S2744-08 PIN photodiode and Hamamatsu S8664-55 (S8148) APD which are commonly used in high energy physics experiments [5-7]. Active areas of the APD and the PIN diode are $5 \times 5 \mathrm{~mm}^{2}$ and $1 \times 2 \mathrm{~cm}^{2}$, respectively.

TABLE I

Radiation damage effects in crystal scintillators [3].

\begin{tabular}{c|c|c}
\hline \hline Crystal properties & PWO & CsI(Tl) \\
\hline color center & yes & yes \\
\hline phosphorescence & yes & yes \\
scintillation & no & no \\
recovery at room temperature & yes & slow \\
dose rate dependence & yes & no \\
thermal annealing yes & no & \\
optical bleaching & yes & no
\end{tabular}

\section{Simulation and results}

The radiation dose in the proposed TAC-PF detector has been simulated through both BESIII Offline Software System (BOSS version 6.6.2) and FLUKA tools $[8,9]$. The BOSS were used to generate an event file from $\mathrm{e}^{-} \mathrm{e}^{+}$ collisions at the center of mass energy of $3.77 \mathrm{GeV}$, which 
is the main framework used in BESIII experiment [10]. The number of expected events is determined multiplying three terms at the designed luminosity level: the cross section of the interested physics process, luminosity and integral of data taking time. Once the FLUKA simulations have been performed for the designed TAC$\mathrm{PF}$ detector, the results have been normalized to evaluate radiation doses by using this factor. The radiation dose level has been expected to be no more than $300 \mathrm{rad} /$ year, which is well below the safety limits. From the FLUKA simulations, it has been foreseen that the integrated radiation dose is about $300 \mathrm{rad}$ over a year operation of the TAC-PF ECAL that is compatible with BESIII detector [11].

The irradiation reduces the crystal transparency without affecting the scintillation mechanism as shown in Fig. $1 \mathrm{a}$ and $\mathrm{b}[12,13]$. PWO crystal partially recovers within a few hours, while $\mathrm{CsI}(\mathrm{Tl})$ crystal recovers slowly in a few weeks after they are removed from the radiation environment.

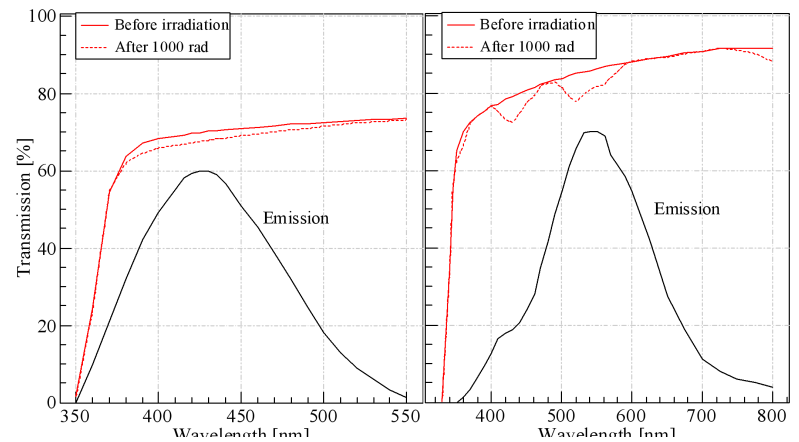

Fig. 1. Emission and transmission spectra of PWO (left) and $\mathrm{CsI}(\mathrm{Tl})$ (right) crystals.

The emission-weighted transmittance $\overline{E W T}$ is given as

$$
\overline{E W T}=\frac{\int T(\lambda) E m(\lambda) \mathrm{d} \lambda}{\int E m(\lambda) \mathrm{d} \lambda},
$$

where $T(\lambda)$ and $E m(\lambda)$ are transmittance and emission of the crystal as a function of wavelength. Its decrease determines the measure of the radiation damage effect on transparency [14].

As a result of the studies on literature and FLUKA simulations that we performed, the radiation dose of $1000 \mathrm{rad}$ causes a decrease in the $\overline{E W T}$ values, which is calculated by using Eq. (1), in rates of $2.78 \%$ and $3.42 \%$ for PWO and CsI(Tl) crystals, respectively, as seen in Table II.

TABLE II

Changes in emission weighted transmittance before and after 1000 rad irradiation

\begin{tabular}{c|c|c}
\hline \hline Crystal type & Before irradiation & After irradiation \\
\hline PWO & 68.96 & 67.04 \\
$\mathrm{CsI}(\mathrm{Tl})$ & 89.05 & 82.18
\end{tabular}

The energy resolution of $a$ calorimeter can be parametrized as $\sigma_{E} / E=a / \sqrt{E} \oplus b \oplus c / E$ where $a$ is the stochastic term, $b$ is the constant term, $c$ is the noise term, $E$ is the incident particle energy in $\mathrm{GeV}$. The stochastic term of the energy resolution for crystal-photodiode combination is quadratic sum of contributions from event to event fluctuations in the lateral shower containment, $a_{\text {lateral }}$, and photoelectron statistics, $a_{p e}$. The contribution from photoelectron statistics is related to fluctuations in the photodiode signal as in the following equation:

$$
a_{p e}=\sqrt{\bar{F} / N_{p e}},
$$

where $\bar{F}$ is the emission weighted excess noise factor coming from the fluctuations in the avalanche gain process. Emission weighted excess noise values for APD structures have been calculated as 2.2 for PWO and 3.8 for $\mathrm{CsI}(\mathrm{Tl})$ crystals in the previous work [4, 15], while there is no internal gain for PIN photodiodes $(F=1)$. $N_{p e}$ is the number of primary photoelectrons resulting in the photoabsorption in the photodiode and it is determined by the multiplication of four terms: coverage of crystal-photodiode, emission weighted transmittance, light yield and emission weighted quantum efficiency [3]. Then, $N_{p e}$ has been calculated for both before and after irradiations by using emission weighted transmittance values given in Table II, the light yields of PWO crystal (120 photon/MeV) [16] and $\mathrm{CsI}(\mathrm{Tl})$ crystal (66000 photon/MeV) [17], experimentally measured emission weighted quantum efficiency values given in [7] and coverage of crystal-photodiode pairs. Consequently, photoelectron statistics contributions to energy resolution are calculated from Eq. (2) as in Table III.

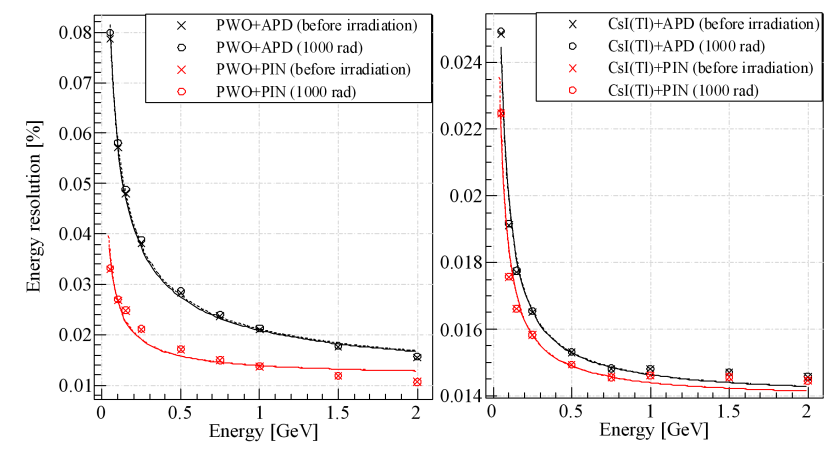

Fig. 2. Energy resolutions as a function of incident energy for both cases, of PWO+photodiode (left) and $\mathrm{CsI}(\mathrm{Tl})+$ photodiode (right), before and after irradiation

Energy resolutions as a function of incident particle energy, as shown in Fig. 2a and b, for different crystal-photodiode pairs have been obtained by adding the photoelectron statistics to the intrinsic energy resolutions, which were obtained from GEANT4 simulations performed in $[4,18]$. When we performed quadratic sum fits, it is noticed that there are no distinguishable changes in the energy resolutions before and after irradiation up to $1000 \mathrm{rad}$. 


\section{TABLE III}

Changes in the photostatistics term, $a_{p e}(\%)$, of the energy resolution for crystal-photodiode combination before and after 1000 rad irradiation

\begin{tabular}{c|c|c}
\hline \hline Crystal+Photodiode & Before irradiation & After irradiation \\
\hline PWO+APD & 1.657 & 1.687 \\
PWO + PIN & 0.444 & 0.453 \\
CsI $(\mathrm{Tl})+\mathrm{APD}$ & 0.242 & 0.246 \\
CsI $(\mathrm{Tl})+\mathrm{PIN}$ & 0.044 & 0.045
\end{tabular}

\section{Conclusion}

In this work, we presented the changes in the stochastic term in the ECAL energy resolutions after irradiation. The radiation levels around proposed TAC-PF ECAL system has been estimated by using FLUKA tool. After a certain radiation dose, the transmission spectra of the proposed PWO and $\mathrm{CsI}(\mathrm{Tl})$ crystals have been slightly reduced. Consequently, insignificant changes have been obtained for photoelectron statistics contributions to the stochastic terms of energy resolution. When we consider PWO and $\mathrm{CsI}(\mathrm{Tl})$ crystals under the dose of $1000 \mathrm{rad}$, it is concluded that a considerable deterioration in energy resolutions of TAC-PF ECAL would not occur for $300 \mathrm{rad}$.

\section{Acknowledgments}

This work was supported by Uludag University Scientific Research Project Unit under project number OUAP(F)-2013/38.

\section{References}

[1] I. Tapan, E. Pilicer, F.B. Pilicer, Nucl. Instrum. Methods Phys. Res. A 831, 389 (2016).

[2] S. Korucuklu, et al., in: Proc. IPAC10, THPD059, (2010).
[3] R.Y. Zhu, in: Handbook of Particle Detection, Ed. C. Grupen, Springer, Berlin 2012, p. 535.

[4] F. Kocak, Nucl. Instrum. Methods Phys. Res. A 787, 144 (2015).

[5] Rihua Mao, Liyuan Zhang, Ren-Yuan Zhu, IEEE Trans. Nucl. Sci. 55, 2425 (2008).

[6] S. Agostinelli, et al., Nucl. Instrum. Methods Phys. Res. A 506, 250 (2003).

[7] R.Y. Zhu, J. Phys. Conf. Series 160, 012017 (2009).

[8] W. Li, et al., in: Proc. CHEP, 2006, p. 225.

[9] A. Ferrari, P.R. Sala, A. Fasso, J. Ranft, FLUKA: a multi-particle transport code, CERN-2005-10, INFN/TC-05/11, SLAC-R-773, (2005).

[10] P.G. Ping, Chin. Phys. C 32, 599 (2008).

[11] M. Ablikim and BESIII Collaboration, Nucl. Instrum. Methods Phys. Res. A 614, 345 (2010).

[12] Rihua Mao, Liyuan Zhang, Ren-Yuan Zhu, IEEE Trans. Nucl. Sci. 59, 2229 (2012).

[13] A. Abashian and BELLE Collaboration, Nucl. Instrum. Methods Phys. Res. A 479, 117 (2002).

[14] Rihua Mao, Liyuan Zhang, Ren-Yuan Zhu, IEEE Trans. Nucl. Sci. 59, 2224 (2012).

[15] E. Pilicer, F. Kocak, I. Tapan, Nucl. Instrum. Methods Phys. Res. A 552, 146 (2005).

[16] R.W. Novotny, W. Doring, K. Mengel, V. Metag, C. Pienne, IEEE Trans. Nucl. Sci. 44, 477 (1997).

[17] C.W.E. van Eijk, Nucl. Instrum. Methods Phys. Res. A 509, 17 (2003).

[18] R.W. Novotny, et al., IEEE Trans. Nucl. Sci. 55, 1283 (2008) 